\title{
Study on Activated Carbon Adsorption of VOCs
}

\author{
Di Zhang ${ }^{1, a}$ \\ ${ }^{1}$ Jilin Business and Technology College, Changchun, Jilin, 130507 \\ ${ }^{\mathrm{a}}$ email
}

Keywords: VOCs, Activated Carbon, Adsorption Character

\begin{abstract}
Toluene, methyl ethyl phthalate amines, methyl ethyl ketone and ethyl acetate are used in China more vinegar several solvents, in the course will emit large amounts of volatile organic gases, serious harm to human health and the environment, it must be recycled or disposed of these gases. In this paper, for several different boiling organic gases: toluene, methyl ethyl phthalate amines, methyl ethyl ketone and ethyl vinegar to study adsorption, desorption and condensation recovery process as well as the law, in order to obtain activated carbon adsorption of pollutants on different boiling points and basic data desorption process, provide a reliable basis for the industrial design of adsorption and desorption processes. Highlights of this paper is to determine the temperature of the adsorption isotherm, domestic normal temperature adsorption studied more, and relatively few of the high temperature adsorption studies, but the adsorption capacity of the activated carbon at high temperature desorption or regeneration of important guiding significance.
\end{abstract}

\section{Introduction}

With industrial development, the application of a very wide range of organic solvents. The main solvent used in paint, oil, paper, printing and chemical industries, the solvent in the course of being out of the volatile organic compounds to the environment and human health are enormous harm. Effective solvent recovery, on the one hand help to reduce production costs, generate economic benefits, but also reduce environmental pollution on the other hand, is an important part of cleaner production. From the perspective of life cycle analysis, solvent recovery also greatly reduce resource solvent production process, energy consumption and environmental pollution, promoting the development of recycling economy and building a sustainable society has a very important practical significance.

\section{Theoretical Basis Adsorption}

Adsorption is a common gaseous pollutants purification method using a porous solid adsorbent gas or liquid mixture of one or several components accumulate or condense on the surface to achieve the purpose of the separation process, especially for treatment of low concentration waste gas high purification is required. Adsorption operation has been widely used in petrochemical, organic chemical production sector has become a major operating unit. In the atmosphere control, because qualitative selective adsorption strong, effective separation is difficult to separate a mixture of other processes and effectively remove low concentrations of toxic and hazardous substances can be widely applied.

Gaseous mixture containing VOCs in contact with the porous solid, the use of solid surface in the presence of unbalanced force of molecular attraction or bonds, the mixed gas VOCs adsorbed component remaining in the solid surface, such a separation process called adsorption control VOCs pollution. Substance is bound to a solid surface is called the adsorbate, adsorbate attached thereto, are known as adsorbents. Depending on the nature between adsorbent and adsorbate adsorption forces, usually divided into physical adsorption and chemical adsorption. Physical adsorption is mainly van der Waals force acting between the molecules, it can be a monolayer, or a multilayer adsorption. Physical adsorption is generally carried out at lower temperatures, in combination with the adsorbent adsorbate weak, reversible process. Usually just raising the temperature, reducing the 
partial pressure of the gas phase adsorbate, adsorbate will precipitate precipitation adsorbate nature has not changed. Therefore, when using physical adsorption, regeneration, the adsorbent adsorbate recovery easier. Physical adsorption amount with the gas temperature drops. With an adsorbent boiling point is proportional to the amount of adsorption of different gases and gas.

By chemisorption bond strength between adsorbent and adsorbate caused in the chemical adsorption process, a chemical reaction between the adsorbent and the adsorbate. It is a selective adsorption of an adsorbent that is only specific adsorption of several substances. Thermal effect of a strong chemical adsorption (adsorption heat is about $84420 \mathrm{~kJ} / \mathrm{mol}$ ), in conjunction with the adsorbent adsorbate is strong, will be desorbed at high temperatures. Chemisorption greater driving force than the physical adsorption, combined with more solid. Therefore, toxic pollutants, chemical adsorption and more reliable.

Can be desorbed molecules are adsorbed to get some energy solid surface, a process known as desorption, adsorption and desorption is the reverse process, under certain conditions, for a sufficient length of time, adsorption and desorption dynamic equilibrium, adsorbent saturation. Adsorbent after saturation, by desorption, adsorption capacity of the adsorbent again. Desorption was desorption process can produce condensate recovery or combustion.

\section{Activated Carbon Adsorption Process of VOCs}

Activated carbon adsorption processes include pressure swing adsorption (PSA), temperature swing adsorption (TAS) and combined with three kinds of TPSA both.

PSA is nearly 50 years developed gas separation, purification and purification technology, thermostat or pyrogen adsorption separation process, the size of the use of the adsorption isotherm slope changes and curvature, change the system pressure on the adsorbate adsorption and desorption. According to the different modes of operation, pressure swing adsorption equilibrium separation type can be divided into two types of separation and speed, respectively depending on the gas adsorption equilibrium differences and differences in performance adsorbents adsorption rate of each component on the adsorbent to achieve gas separation. The Act cycle operation can be achieved with a high degree of automation, low investment, low energy consumption, safety advantages. United States, Britain and other countries initially used legal pressure swing adsorption $\mathrm{H} 2$, N2, and O2, extracting CO2, CO [2]. After the 1960s, it began to use light hydrocarbon separation and recovery of the law. Bochum University in Germany and other RÊhm activated carbon C40/4 pressure swing adsorption recovery $\mathrm{CH} 2 \mathrm{Cl} 2$ and toluene recoveries were $80 \%$ and 95\%, using Wessalith/DAY xylene recovery, and the recovery of more than 95\% [3]. ReikoWakasugi, who used enriched reflux recovery of ethanol gas pressure swing adsorption system [4], Liu and other activated carbon pressure swing adsorption recovery of benzene from benzene/nitrogen gas mixture, the recovery rate of 99\% [5]. Olajossy et al vacuum pressure swing adsorption (VPSA) The coal mine gas methane volume concentration increased from 5512\% to 86\% to 91\%. German company Bayer using D47/4 activated carbon pressure swing gas separation acetone recovery rate of 95\%. Japan Bell company pressure swing adsorption method using activated charcoal recovered purity of $98 \%$ ethanol products. Gas separation Mechanical Engineering Research Institute, Shanghai Research Institute of Beijing University of Science and Technology have conducted research of pressure swing adsorption VOCS. China also has a number of enterprises to adopt pressure swing adsorption recovery VOCS, such as: Xinjiang Tianye recall of vinyl chloride and acetylene, Jingmen Petrochemical Plant a catalytic ethylene plant recovery, Sichuan Tianyi Science and Technology Co., Ltd. and Taiyuan Chemical Industry Co., Ltd. jointly developed PSA recycling of PVC vinyl chloride distillation tail gas, acetylene technology, stable operation, good effect.

Since the pressure swing adsorption process requires constant pressure, reduced pressure or vacuum, pressure control and vacuum pressure pumping system, pay attention to the dead space gas pressure, complex operation, huge energy consumption, and thus the current use of temperature swing adsorption, temperature fixed bed adsorption again mostly because of the high fixed bed adsorption efficiency, simple equipment, technology is relatively mature. 


\section{Adsorption Character of Activated Carbon}

Researches of activated carbon adsorption law will be more, and especially more indepth study abroad, but because there is a big difference in the nature of the activated carbon itself at home and abroad, and therefore not suitable for direct overseas base data used in the domestic activated carbon, and At present, the basic data of polluting gases adsorbed aspects related literature few reports, so this issue of great significance. This paper studies the law of activated carbon adsorption, mainly on a few select industrial applications more widely and have different boiling point and different polarity of volatile organic compounds, as chemisorption, and choose to use more coal quality activated carbon as an adsorbent in the industry, research activated carbon fixed bed adsorption process dynamic some variation.

Experiment segmented filled with activated carbon adsorption dynamically generated organic gas bubbling in the glass adsorption column, the working process of the experimental apparatus is as follows: 2 valves regulate air pump 1 bulged, bulging air is divided into two, with 3 and 4 to adjust the flow meter and metering, all the way to the smaller air flow into the gas generating bottles 5 . Gas generating bottle containing an organic solvent, a thermostat water bath 6 , adjust the temperature water bath temperature can be controlled amounts of volatile organic solvents. After the occurrence of air into the bottle, the amount of organic solvent vapors out, mixed with air large flow the other way, resulting in a concentration of organic gases. The organic flow of gas measured by the flow meter 8, the concentration is measured by a detector PD into three sessions of activated carbon adsorption column filled with glass after $\mathrm{n}$ emissions. Taking into account the experimental device inlet concentration need to set debug bypass discharge port switching valves 9 and 10 are controlled by the discharge port. Further, taking into account the dynamic bubbling concentration of the organic gas produced may be unstable, so the gas generating bottle after adding a buffer gas bottle 7, the concentration of the organic gas is more stable.

Specifications small glass adsorption column $30 \sim$ x1200 , adsorption column is divided into three sections, each separated by a stainless steel wire mesh and stainless steel mesh as a support, activated carbon and then fill about 129 in each of the stainless steel wire mesh, glass adsorption column has four gas sampling ports, respectively, an intake port or an organic gas sampling and sampling ports three segments.

In the normal temperature adsorption of small scale experiments, activated carbon adsorption capacity is determined mainly weighing method. Before and after weighing the quality of the activated carbon adsorption of organic gas concentration according to time and each outlet, the curve drawn through the organic gas, and then calculate the height of the adsorbent bed must be in accordance with the breakthrough time breakthrough curves and adsorption saturation time the height of the mass transfer zone, by contrast curve penetrating study of the different conditions of different adsorption adsorbate mass transfer law.

In this study, Tangshan Jianxin Activated Carbon Co., Ltd. of JX400 series of coal activated carbon adsorption toluene (nonpolar, boiling point), butanone (less polar, lowboiling) and dimethyl amine acetate phthalate (polar, high boiling point) of these three volatile organic compounds, those of the different adsorption studies inlet concentrations, gas flow rate and the filling height of the carbon bed operating conditions, including the shape of the curve and through the breakthrough time, the adsorption capacity is saturated, the mass transfer zone height. Adsorbed on activated carbon adsorption experiments glass column, you can get three sections with different filling height of breakthrough curves, this law can be analyzed for different activated carbon adsorption of VOC.

In certain inlet concentration and flow rate, with the increase of the thickness of the bed, breakthrough curves of substantially the same shape, the center of the adsorption mass transfer zone is equal to the slope. Activated carbon bed depth affects only reach saturation adsorption breakthrough time and duration of action, because it is at a certain velocity and the inlet concentration, the adsorption mass transfer rate constant, the adsorption mass transfer zone at the same height, so the bed depth is only change the arrival time and the protective effect of the penetration time. That is when the adsorption temperature is constant and the gas inlet concentration and flow rate constant, highly activated carbon bed can only make the breakthrough curve 
substantially parallel to move around.

At the same flow rate, inlet concentration, the more readily activated carbon layer is penetrated; import greater the concentration, the greater the adsorption of the center of the slope of the mass transfer zone, i.e. the adsorption mass transfer faster, easier reach adsorption equilibrium saturation, saturation the shorter duration of action. By calculating the adsorption capacity of different inlet concentrations it can be known, the greater the inlet concentration, the greater the activated carbon adsorption capacity of toluene. That is at the same inlet concentration, gas flow rate is increased to make the breakthrough curve steepening, adsorption mass transfer zone center slopes increase the penetration time and saturation time forward. This may be because the flow rate of the gas in the acceleration of the tower, adsorbate residence time in the column becomes shorter, with activated carbon is insufficient, so that the penetration time is shorter, but also absorb the mass transfer zone becomes long.

At the same flow rate as toluene inlet concentration increases, the breakthrough time will be shortened, the height of the mass transfer zone becomes long, and activated carbon adsorption capacity increases. At the same concentration of imports, increased imports of gas flow, that is, increase the air speed, the activated carbon adsorption of toluene breakthrough time will be shortened, and the height of the mass transfer zone length, but the activated carbon adsorption capacity will be reduced.

\section{Conclusion}

As noted by the US EPA, activated carbon adsorption technology is the best that can be employed to remove VOCS. Especially activated carbon fixed bed adsorption temperature swing regeneration techniques suitable for our current economic, technological level, and high purity recovered, will become the mainstream of future development. Design and manufacture of new types of activated carbon adsorption with better performance or to meet specific needs, to find effective methods of surface modification of activated carbon; strengthen the study of activated carbon adsorption process factors and improve the adsorption efficiency; strengthen computer simulation VOCS adsorptive separation processes and intelligent control, continuous operation of adsorption and desorption of the recent major research topic.

\section{References}

[1] Reiko Wakasug, i Akio Kodama, Motonobu Goto, etal1Recovery of Volatile Organic Compounds as Condensate by Pressure Swing Adsorption with Enriching Reflux [J]. Journal of Chemical Engineering of Japan, 2004, 37(2): 374377.

[2] Yujun Liu, JamesA Ritter, BalK Kaul. Simulation of gasoline vapor recovery by pressure swing adsorption [J]. Separation and Purification Technology, 2000, 20(1): 111127.

[3] Olajossy A,Gawdzik A, BudnerZ, etal1Methane separation from coal mine methane gasby vacuum pressure swing adsorption [J].Chemical engineering research \& design, 2003, 81(4): 474482.

[4] LoukidouM X,ZouboulisA I1Comparison of two biological treatment processes using attached growth biomass for sanitary land fill leachate treatment [J]. Environmental Pollution, 2001, 111(2): 273281.

[5] JeongHo Yun, Daeki Cho,i HeeMoon1Benzene adsorption andhotpurge regeneration in activated carbon beds [J]. Chemical Engineering Science, 2000, 55(23): 58575872.

[6] ByungMan Jeong, EuiSubAhn, Jeong Hoyun, etal1Ternary adsorption equilibrium of H2/CH4/C2H4onto activated carbon [J]. Separation and Purification Technology, 2007, 55(3): 335342. 